Marquette University

e-Publications@Marquette

$11-1-2015$

\title{
Pain Response after Maximal Aerobic Exercise in Adolescents across Weight Status
}

Stacy Stolzman

Marquette University

Michael E. Danduran

Marquette University, michael.danduran@marquette.edu

Sandra K. Hunter

Marquette University, sandra.hunter@marquette.edu

Marie K. Hoeger Bement

Marquette University, mariehoeger.bement@marquette.edu

Accepted version. Medicine \& Science in Sports \& Exercise, Vol. 47, No. 11 (November 2015):

2431-2440. DOI. (C) 2015 American College of Sports Medicine. Used with permission. 


\title{
Pain Response after Maximal Aerobic Exercise in Adolescents across Weight Status
}

\author{
Stacy Stolzman
}

Clinical and Translational Rehabilitation Health Sciences, Department of Physical Therapy, Marquette University,

Milwaukee, WI

Michael Danduran

Program in Exercise Science, Department of Physical Therapy,

Marquette University, Milwaukee, WI

Herma Heart Center, Children's Hospital of Wisconsin,

Milwaukee, WI

Sandra K. Hunter

Clinical and Translational Rehabilitation Health Sciences, Department of Physical Therapy, Program in Exercise Science,

Department of Physical Therapy, Marquette University,

Milwaukee, WI

Marie Hoeger Bement

Clinical and Translational Rehabilitation Health Sciences, Department of Physical Therapy, Marquette University,

Milwaukee, WI 
NOT THE PUBLISHED VERSION; this is the author's final, peer-reviewed manuscript. The published version may be accessed by following the link in the citation at the bottom of the page.

\section{Abstract}

Introduction: Pain reports are greater with increasing weight status, and exercise can reduce pain perception. It is unknown, however, whether exercise can relieve pain in adolescents of varying weight status. The purpose of this study was to determine whether adolescents across weight status report pain relief after high-intensity aerobic exercise (exerciseinduced hypoalgesia (EIH)).

Methods: Sixty-two adolescents $(15.1 \pm 1.8 \mathrm{yr}, 29$ males) participated in the following three sessions: 1) pressure pain thresholds (PPT) before and after quiet rest, clinical pain (McGill Pain Questionnaire), and physical activity levels (self-report and ActiSleep Plus Monitors) were measured, 2) PPT were measured with a computerized algometer at the fourth finger's nailbed, middle deltoid muscle, and quadriceps muscle before and after maximal oxygen uptake test ( $\mathrm{V} \cdot \mathrm{O}_{2 \max }$ Bruce Treadmill Protocol), and 3 ) body composition was measured with dual-energy $\mathrm{x}$-ray absorptiometry.

Results: All adolescents met criteria for $\mathrm{V} \cdot \mathrm{O}_{2 \max }$. On the basis of body mass index z-score, adolescents were categorized as having normal weight ( $\mathrm{n}=$ 33 ) or being overweight/obese $(n=29)$. PPT increased after exercise (EIH) and were unchanged with quiet rest (trial $\times$ session, $P=0.02$ ). EIH was similar across the three sites and between normal-weight and overweight/obese adolescents. Physical activity and clinical pain were not correlated with EIH. Overweight/obese adolescents had similar absolute $\mathrm{V} \cdot \mathrm{O}_{2 \max }\left(\mathrm{L} \cdot \mathrm{min}^{-1}\right)$ but lower relative $\mathrm{V} \cdot \mathrm{O}_{2 \max }\left(\mathrm{mL} \cdot \mathrm{kg}^{-1} \cdot \mathrm{min}^{-1}\right)$ compared with normal-weight adolescents. When adolescents were categorized using FitnessGram standards as unfit $(n=15)$ and fit $(n=46)$, the EIH response was similar between fitness levels.

Conclusions: This study is the first to establish that both overweight and normal-weight adolescents experience EIH. EIH after high-intensity aerobic exercise was robust in adolescents regardless of weight status and not influenced by physical fitness.

Key Words: Pain Relief, Children, Body Mass Index, V·O2max, Physical Activity, Physical Fitness

Exercise can decrease pain (i.e., exercise-induced hypoalgesia $(\mathrm{EIH}))$ in adults and is dependent on both the intensity and duration of the exercise stimulus. ${ }^{19}$ To maximize EIH, aerobic exercise should be performed at a moderate/high intensity and for longer duration. ${ }^{19,39} \mathrm{EIH}$ is systemic in that pain relief is not localized to the exercising muscle, although some studies show that EIH is more robust in the exercising body part compared with nonexercising body parts. ${ }^{20,39}$ All of these studies have been conducted on adults. To our knowledge, there are no studies investigating the effect of exercise on pain perception in adolescents. Identifying the EIH response in

Medicine \& Science in Sports \& Exercise, Vol 47, No. 11 (November 2015): pg. 2431-2440. DOI. This article is @ American College of Sports Medicine and permission has been granted for this version to appear in e-Publications@Marquette. American College of Sports Medicine does not grant permission for this article to be further copied/distributed or hosted elsewhere without the express permission from American College of Sports Medicine. 
pediatric populations will help establish the potential benefits of exercise as a nonpharmacological pain management tool.

Weight status is an understudied factor in pain perception. Of the few studies, obese adults have lower pain sensitivity to a noxious stimulus (higher pain thresholds) compared with nonobese adults. 7,30 In contrast, clinical pain reports tend to increase as the weight status of both adults and adolescents increases. ${ }^{27,33}$ Unfortunately, pain is commonly overlooked as a health outcome despite most obese youth reporting that they currently feel pain. ${ }^{14}$ Physical and psychosocial consequences of obesity include development of musculoskeletal dysfunction, poor quality of life, missed school, and social withdrawal. ${ }^{13,17}$ Thus, strategies to manage pain in obese adolescents are important for quality of life and health.

Physical inactivity is a major contributor to the obesity epidemic $\underline{22}$ and is associated with the increase in pain reported in this population. ${ }^{33}$ Pediatric barriers to participation in physical activity include age (adolescents are at greater risk than younger children), ${ }^{10}$ weight status (obese youth are at greater risk than normal-weight youth), ${ }^{10}$ and presence of pain. ${ }^{24}$ Thus, overweight adolescents with pain may be at high risk for sedentary behavior, resulting in poor physical fitness levels. Finally, self-reported physical activity is associated with endogenous pain modulation; ${ }^{23}$ individuals that report higher levels of physical activity have more efficient descending pain modulatory function. Furthermore, several chronic pain conditions (e.g., fibromyalgia, irritable bowel syndrome, headache, etc.) often exhibit decreased endogenous pain modulation. ${ }^{31}$ Because endogenous pain modulation is one of the mechanisms for pain relief associated with exercise, individuals that are more physically active may experience greater reduction in response to a pain stimulus with exercise (i.e., EIH) whereas individuals that report current pain may experience less EIH.

Understanding how pain changes with exercise will provide a scientific rationale to better use exercise in the management of pain in pediatric populations. The purpose of this study was to compare the magnitude of EIH in normal-weight and overweight/obese adolescents after a single session of intense aerobic exercise. To determine the possible modifying effect of baseline fitness, we also compared EIH in adolescents of varying levels of physical fitness in both normal-weight 
and overweight/obese adolescents. We hypothesized that normalweight and overweight/obese adolescents would experience similar levels of EIH but those who were more physically fit would experience greater EIH. Because physical activity levels, presence of pain, and psychosocial influences (i.e., pain catastrophizing and quality of life) have been implicated in pain modulation, we examined their influence on EIH in the adolescents.

\section{METHODS}

\section{Subjects}

Sixty-two adolescents (15.1 $\pm 1.8 \mathrm{yr}(12.0-17.9 \mathrm{yr}) ; 29$ boys and 33 girls) and their parent/legal guardian were recruited from a Midwestern United States metropolitan area (Milwaukee, WI) through community flyers, the Marquette University electronic newsletter, a monthly parenting magazine advertisement, and a Facebook advertisement. The adolescents were enroled as part of a larger study that investigated the association among inflammatory markers, physical fitness, and pain in adolescents of varying weight status. All adolescents were screened via phone conversation with a parent/legal guardian about the study components and exclusionary criteria. Adolescents in good health were eligible for participation in the study, with the following exclusions: 1 ) body mass index (BMI) below the 10th percentile for age and sex, 2) inability to report pain threshold (i.e., tissue trauma or neurological condition that would affect sensory perception), 3) unable to tolerate ice water submersion (e.g., Raynaud disease or cold urticaria), 4) exercise contraindications, 5) non-English speaking participants, 6) cognitive delays, 7) pregnancy, 8) claustrophobia, or 9) history of mental health disorder. The protocol was approved by the institutional review board at the Marquette University.

\section{Experimental Protocol}

Adolescents participated in three experimental sessions with approximately $1 \mathrm{wk}$ between sessions. The respective parent/legal guardian completed questionnaires related to the adolescent's health and wellness. At the start of the first session, the adolescent and parent completed the assent and consent, respectively. During this session, resting vitals, weight status, experimental pain (i.e., pressure pain thresholds (PPT)) before and after 20 min of quiet rest, clinical 
pain, and self-report physical activity levels were measured.

Adolescents were also instructed on wearing the physical activity monitors. After the first session, adolescents participated in either the treadmill or dual-energy $x$-ray absorptiometry (DXA) sessions in a counterbalanced manner. The treadmill session involved the measurement of experimental pain before and after a maximal aerobic treadmill test (maximal oxygen uptake test $\left(\mathrm{V} \cdot \mathrm{O}_{2 \max }\right)$ ) along with psychosocial assessments (i.e., quality of life and pain catastrophizing). The DXA session measured body composition.

\section{Weight Status and Body Composition}

From height $(\mathrm{cm})$ and mass $(\mathrm{kg})$ measurements, BMI was calculated and plotted for percentiles and z-scores. On the basis of BMI z-scores, 33 adolescents were classified as having normal weight (BMI z-score < 1.00), and 29, as overweight/obese (BMI z-score > = 1.00). ${ }^{29}$

Total body DXA scans were performed with the Lunar GE Prodigy (GE, Madison, WI) bone densitometer to determine body composition. Before each scan, a quality assurance protocol was completed, a phantom was scanned for calibration purposes, and female adolescents completed a pregnancy test. Scans were analyzed using the Lunar GE Prodigy pediatric software to quantify fat body mass $(\mathrm{kg})$, lean body mass $(\mathrm{kg})$, total body fat (\%), android fat (\%), gynoid fat (\%), and android/gynoid (A/G) ratio. Adolescents were classified as either android $(A / G$ ratio $>=1.0$ ) or gynoid $(A / G$ ratio < 1.0). Total body fat $z$-scores for age and sex were determined using the online Baylor College of Medicine Body Composition Laboratory Pediatric Body Composition Reference Charts. ${ }^{18}$

\section{Experimental Pain-PPT Testing}

PPT were measured during the first experimental session (before/after 20-min quiet rest) and before/after maximal aerobic exercise (treadmill session) at three sites: left fourth finger's nailbed, left middle deltoid muscle (one-quarter the distance from the acromion to the lateral epicondyle), and right quadriceps muscle (half the distance from the anterior superior iliac crest to the superior patella). $\underline{2}$ Three trials were completed at each site with a 10-s interstimulus interval, and the site order was randomized at each session. A battery-operated pressure algometer (Algomed) with a 1- 
$\mathrm{cm}^{2}$ probe was placed on each site at a rate of $50 \mathrm{kPa} \cdot \mathrm{s}^{-1} .{ }^{16}$ The adolescents were seated and instructed to push the patient response unit when they first felt pain (i.e., pain threshold), which was electronically recorded in kilopascals.

\section{Pain and Quality of Life Assessments}

\section{McGill questionnaire (MPQ)}

Participants completed the MPQ during the first session. This questionnaire evaluates the multidimensional aspect of pain (sensory, affective, and cognitive) related to current pain. Higher scores represent more pain.

\section{Pain catastrophizing scale-child and parent (PCS-C and PCS-P)}

To address pain catastrophizing (i.e., negative mental response to anticipated or actual pain), the adolescent and respective parent completed the PCS-C and PCS-P during the treadmill session. The questionnaire has 13 statements that are scored in a Likert Scale (05) with three subcategories: rumination, magnification, and helplessness. Higher PCS scores are indicative of higher pain catastrophizing with clinical reference points for the PCS-C as low (014), moderate (15-25), and high (>26). ${ }^{28}$

Pediatric quality of life inventory (PedsQL)

All adolescents and respective parents completed the PedsQL for child (8-12 yr of age) or teen (13-18 yr of age) with the corresponding parent version. The PedsQL is a valid and reliable measurement of pediatric health-related quality of life and has been used in youth of varying weight status. ${ }^{40}$ The PedsQL was completed during the treadmill session and was scored for total, physical, social, emotional, and school domains. Higher PedsQL scores represent higher quality of life. Total PedsQL cutoff scores for impaired quality of life are 69.7 for the child/teen and 65.4 for the parent. ${ }^{40}$

\section{Physical Activity Assessments}

Physical activity was quantified with accelerometers and selfreported physical activity levels. Adolescents were instructed to wear the ActiGraph monitor (ActiSleep Plus Monitor, Pensacola, FL) ${ }^{9}$ on the nondominant wrist for seven consecutive days, complete daily logs (awake/sleep times, physical activity participation, and removal/reapplication of the device), and return the device at session

Medicine \& Science in Sports \& Exercise, Vol 47, No. 11 (November 2015): pg. 2431-2440. DOI. This article is (C) American College of Sports Medicine and permission has been granted for this version to appear in e-Publications@Marquette. American College of Sports Medicine does not grant permission for this article to be further copied/distributed or hosted elsewhere without the express permission from American College of Sports Medicine. 
2. Data were downloaded via the ActiLife 6 Data Analysis Software (ActiGraph, Pensacola, FL) at 60-s epochs. Wear time validation was done, ${ }^{4}$ and adolescents were included in data scoring if they met the youth wearing recommendations for ActiGraph monitors (at least one weekend day and four weekdays for $\left.>950 \mathrm{~min} \cdot \mathrm{d}^{-1}\right){ }^{3} \mathrm{Next}$, the data were scored using pediatric cutoffs ${ }^{11}$ to quantify length of time (min) in sedentary activity, light activity, and moderate/vigorous activity along with vector magnitude counts per minute and step counts. Additional sedentary analysis was completed to determine the average length of sedentary bouts ( $\mathrm{min}$ ), as defined by $>=10 \mathrm{~min}$ with $<=99$ counts per minute. ${ }^{37}$ Wear time of the ActiGraph monitor was compared with the written activity logs completed by each adolescent.

Self-reported physical activity was quantified using the Physical Activity Questionnaire-Elementary School and High School Versions (PAQ). The PAQ is a reliable and valid instrument that provides a general measure of physical activity for youth from grades 4 to 12 (approximate age of 8-20 yr). Higher PAQ scores represent higher general physical activity. Cutoff points have been proposed to categorize youth as "at risk" or "no risk" for metabolic syndrome. Cutoff points for "at risk" are $<2.9 / 5$ for boys and $<2.7 / 5$ for girls. ${ }^{41}$

\section{Maximal Aerobic Treadmill Test ( $\left.\mathrm{V} \cdot \mathrm{O}_{2 \max }\right)$}

Adolescents completed a maximal aerobic treadmill test ( $T$ 2100 Treadmill; GE Healthcare, EI Paso, TX) with a V $\cdot \mathrm{O}_{2 \max }$ Bruce protocol, which involved an increase in the grade and speed of the treadmill every 3 min. ${ }^{8}$ Twelve-lead EKG (CASE Cardiosoft V6.61; GE Healthcare, El Paso, TX) was obtained, and metabolic monitoring (Encore 29c; VMAX, Palm Springs, CA) of expired gases $\left(\mathrm{O}_{2}\right.$ and $\left.\mathrm{CO}_{2}\right)$ and volumes were measured continuously online with 20 -s averaging. Variables assessed from the $\mathrm{V} \cdot \mathrm{O}_{2 \max }$ protocol include peak respiratory rate $(R R)$, relative $\mathrm{V} \cdot \mathrm{O}_{2 \max }\left(\mathrm{mL} \cdot\right.$ total body mass $\left.\mathrm{kg}^{-1} \cdot \mathrm{min}^{-1}\right)$, and absolute $\mathrm{V} \cdot \mathrm{O}_{2 \max }\left(\mathrm{L} \cdot \mathrm{min}^{-1}\right)$. Lean $\mathrm{V} \cdot \mathrm{O}_{2 \max }\left(\mathrm{mL} \cdot\right.$ lean body mass $\mathrm{kg}^{-1} \cdot \mathrm{min}^{-}$ ${ }^{1}$ ) was also calculated by dividing absolute $\mathrm{V} \cdot \mathrm{O}_{2 \max }$ by the lean body mass $(\mathrm{kg}){ }^{21}$ the lean body mass was obtained from the DXA scan.

Adolescents reported RPE (0-10) at the end of each 3-min stage and at termination. Verbal encouragement was given throughout the test until the subject signaled that they wanted to terminate the test. Criteria for establishing $\mathrm{V} \cdot \mathrm{O}_{2 \max }$ was based on 
meeting at least two of the following four criteria: 1) volitional fatigue $(\mathrm{RPE}>8), 2)$ respiratory quotient $(\mathrm{RQ})>1.0,3) \mathrm{HR}>85 \%$ agepredicted $\mathrm{HR}_{\max }{ }^{42}$ and 4) plateau in $\mathrm{O}_{2}$ consumption. Upon completion of the treadmill test, recovery included walking ( $2 \mathrm{~min}$ ) followed by sitting ( $2 \mathrm{~min}$ ). Immediately after the 4-min recovery, measurement of postexercise experimental pain (PPT) was completed.

Adolescents were categorized as "fit" or "unfit" according to their relative $\mathrm{V} \cdot \mathrm{O}_{2 \max }\left(\mathrm{mL} \cdot \mathrm{kg}^{-1} \cdot \mathrm{min}^{-1}\right)$ and FitnessGram standards. Specifically, the FitnessGram has established criteria (age and sex specific) to classify a youth's aerobic capacity $\left(\mathrm{mL} \cdot \mathrm{kg}^{-1} \cdot \mathrm{min}^{-1}\right)$ as being in the healthy fitness zone. This threshold for aerobic capacity, which varies according to age and sex, represents the minimum fitness level to offer protection against diseases that result from sedentary living. ${ }^{34}$ For example, the healthy fitness zone for a $12-y r-$ old female is $>=40.1 \mathrm{~mL} \cdot \mathrm{kg}^{-1} \cdot \mathrm{min}^{-1}$, whereas the healthy zone for a 17 -yr-old male is $>=44.2 \mathrm{~mL} \cdot \mathrm{kg}^{-1} \cdot \mathrm{min}^{-1}$. Using these $\mathrm{V} \cdot \mathrm{O}_{2 \max }$ criteria, each adolescent was categorized into either the "fit" group (within the healthy fitness zone) or the "unfit" group (below the healthy fitness zone).

\section{Statistical Analysis}

Data were analyzed using Statistical Package for the Social Sciences (SPSS version 21; IBM, Chicago, IL) and reported as mean $\pm S D$ within the text and table and the mean \pm SE in the figure. A $P$ value $<=0.05$ was used for statistical significance.

PPT was calculated by averaging the three thresholds at each site (nailbed, deltoid muscle, and quadriceps muscle). Mixed-design multivariate repeated-measures ANOVA was used to assess for change in PPT (trial (before and after) $\times$ session (baseline and treadmill) $\times$ site (nailbed, deltoid muscle, and quadriceps muscle)). Weight status (normal weight or overweight/obese), fitness level (fit or unfit), and body composition (android or gynoid) were betweensubject factors. When a significant effect was found, Bonferronicorrected t-tests for post hoc multiple comparisons were used to identify differences. Pearson correlations were calculated to determine associations between EIH (before/after PPT at combined sites) and dependent variables. 
RPE during the treadmill test was analyzed using repeatedmeasures ANOVA (time: end of stage 1, midpoint, and termination) using between-subject factors of weight status (time $\times$ weight status (normal weight or overweight/obese)) and fitness levels (time $x$ fitness levels (fit or unfit)). Post hoc independent t-tests were used when appropriate. Independent t-tests were completed between the weight (normal weight or overweight/obese) and fitness (fit or unfit) groups to identify potential differences in demographics, weight and body composition, physical activity, physical fitness, health, pain (MPQ, present pain intensity, and baseline (preexercise) PPT), and psychosocial measures. Additional independent t-tests were used to compare adolescents at risk and adolescents at no risk for metabolic syndrome.

Physical activity monitor data were analyzed via the ActiLife 6 Data Analysis Software (ActiGraph, Pensacola, FL) (Fig. 1). Twelve subjects were not included in wear time analysis because of the following: 1 ) loss of monitor ( $n=4$; one normal-weight fit, one overweight/obese fit, and two overweight/obese unfit adolescents), 2) refusal to wear the monitor for the full duration ( $n=4$; two normalweight fit and two overweight/obese fit adolescents); and 3) choice of not wearing the monitor because of participation in organized swimming or baseball regulations ( $n=4$; all normal-weight fit adolescents). Data from 50 adolescents underwent ActiLife pediatric wear time validation, ${ }^{4}$ and four adolescents were excluded because they did not wear the device for the minimum time. ${ }^{3}$ Final data scoring from 46 adolescents ( $n=27$ normal weight, 23 fit and two unfit; and $n=19$ overweight/obese, 12 fit and seven unfit) was completed to quantify levels of physical activity. ${ }^{11}$ Of the 46 adolescents, 11 adolescents ( $n=6$ normal weight, five fit and one unfit; and $n=5$ overweight/obese, four fit and 1 unfit) were required as per coach/referee rules to remove the device during practices and/or competitive sporting events. 
NOT THE PUBLISHED VERSION; this is the author's final, peer-reviewed manuscript. The published version may be accessed by following the link in the citation at the bottom of the page.

\section{Results}

\section{Figure 1}

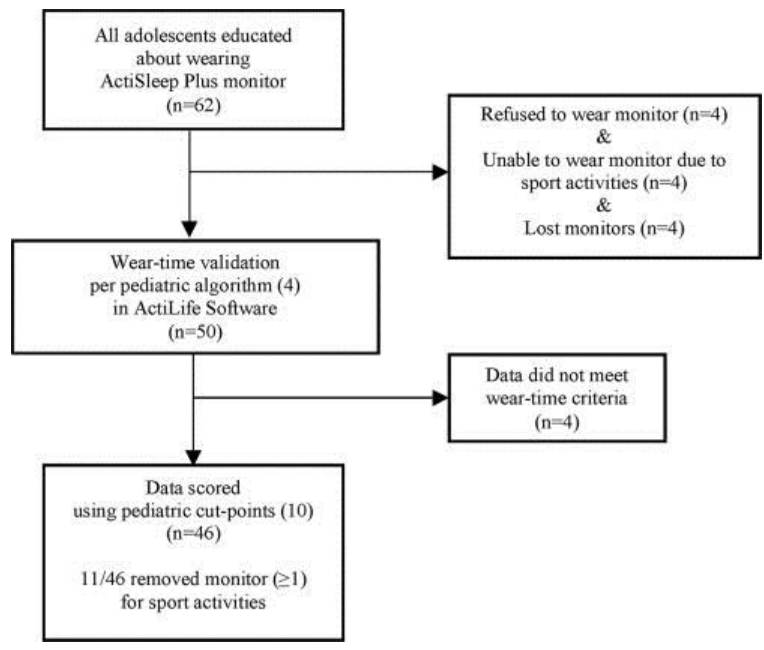

\section{Subjects}

Of the 62 adolescents (and respective parent/legal guardian) that participated in this study, one adolescent was excluded from each of the $\mathrm{EIH}, \mathrm{DXA}$, and $\mathrm{V} \cdot \mathrm{O}_{2 \max }$ analyses because of technical difficulties with the software, positive pregnancy test result, and operational error, respectively.

\section{Baseline Measures in Normal-Weight versus Overweight/Obese Adolescents}

On the basis of the BMI z-score classification, 33 adolescents were of normal weight and 29 were overweight/obese (Table 1). Overweight/obese adolescents had higher total body fat percentage, total body fat $z$-score, and fat mass (all $P<0.0001$ ) but similar lean body mass $(P>0.05)$ compared with normal-weight adolescents. All adolescents were classified as either gynoid $(n=41 ; 18$ males and 23 females; 31 of normal weight, 10 overweight/obese) or android ( $n$ $=20 ; 11$ males and nine females; two of normal weight, 18 overweight/obese) on the basis of the DXA scan. 
NOT THE PUBLISHED VERSION; this is the author's final, peer-reviewed manuscript. The published version may be accessed by following the link in the citation at the bottom of the page.

\begin{tabular}{|c|c|c|c|c|}
\hline & Normal Weight $(a=83)$ & Oveweighthotese $(n=29)$ & FII $(a=45)$ & Uaflit $(a=15)$ \\
\hline \multicolumn{5}{|l|}{ Dengraghics } \\
\hline Sex (mabs) & 17 & 12 & 23 & 6 \\
\hline \multirow{2}{*}{\multicolumn{5}{|c|}{ Elrnich }} \\
\hline & & & & \\
\hline Coucrglan & 31 & 16 & 40 & 6 \\
\hline Africas Amaricas & 0 & 9 & 3 & 6 \\
\hline Hspanic & 2 & 4 & 3 & 3 \\
\hline Vitas & $n=33$ & $n=29$ & $n=46$ & $\pi=15$ \\
\hline Resting HR $\{t o n\}$ & $72.5 \times 11.4$ & $780+119$ & $71.7+11.3$ & $85.5+70^{\circ}$ \\
\hline Aessing systolic BP (mm Hq) & $167.1+107$ & $115.7+11.3 *$ & $1004+11.7$ & $116.8+10.6 \cdots$ \\
\hline Resing dastolis BP (mm th) & $71.3=6.8$ & $74.7 \pm 8.7$ & $71.6=69$ & $76.5 \pm 88^{*} \cdots$ \\
\hline Resting MuP (min Ha) & $33.2=6.4$ & $879+8.6 \cdots$ & $342+70$ & $900 \pm 8.6 \cdots$ \\
\hline Pesing palas ex fst & $\$ 7.3=1.1$ & $975+1.1$ & $97.4 \pm 12$ & $97.3+0.9$ \\
\hline Weight stuus and body comsosition & $n=33$ & $n=28$ & $n=46$ & $a=14$ \\
\hline $\operatorname{eM}\left(\mathrm{og}^{-2}\right)$ & $21.1+1.9$ & $305 \pm 6.8^{\circ}$ & $23.2+3.4$ & $33.4+8.4^{*}$ \\
\hline EMI zemare & $0.27 \pm 0.47$ & $1.87 \pm 0.50^{\circ}$ & $0.72 \pm 0.75$ & $2.04 \div 0.64^{\circ}$ \\
\hline Total toby tas (s) & $209=92$ & $40.9 \pm 76^{\circ}$ & $26.7=11.9$ & $41.8 \div 10.2$. \\
\hline Total bosy fas zecone fare and sex) & $-6.00=1.00$ & $1.00 \pm 0.47^{\circ}$ & $-0.05 \div 1.34$ & $1.58=0.85$. \\
\hline Androd bat ist & $223=11.5$ & $47.1 \pm 8.6$ & $29.6+14.6$ & $46.3=12.2$ \\
\hline Gmoid tat (S) & $290=10.7$ & $46.6 \pm 6.7 \%$ & $34.4+12.4$ & $463=18^{\circ}$ \\
\hline NG rato & $0.74=0.19$ & $10 \div 0.11$. & $0.82 \times 0.19$ & $103 \div 0.16^{\circ}$ \\
\hline Leas body mos (ky) & $45.40=10.56$ & $47.61=12.23$ & $45.22+10.50$ & $51.48 \div 13.06$ \\
\hline Fac boty mass avo & $11.91=5.52$ & $33.90 ; 13.39^{\circ}$ & $16.88 \times 0.16$ & $3956 \div 16.81$. \\
\hline \multicolumn{5}{|l|}{ Ptresca actwity } \\
\hline Setl-reparted flirsical actiaty & $n=33$ & $n=29$ & $n=46$ & $a=15$ \\
\hline PAO SOONE (0-5) & $27 \times 0.6$ & 25.67 & $27+0.6$ & $23 \times 07 \cdots$ \\
\hline Actisnoh accederometry & 8.26 & $0=20$ & $a=35$ & $t=10$ \\
\hline Averose lasgh of sodemey bouts imin) & $228+27$ & $25.1+20^{\circ}$ & $233 \pm 27$ & $256 \times 17 \cdots$ \\
\hline Sedosary on & $537 \div 56$ & $54.15 \pm 5.7$ & $534 \pm 5.6$ & $54.9+5.6$ \\
\hline Lote activen (s) & $11.6=1.6$ & $119=14$ & $11.6+1.5$ & $12.3+1.6$ \\
\hline 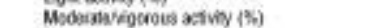 & $347+53$ & $34.0 \times 5.7$ & $35.0+5.4$ & $328 \times 54$ \\
\hline \multirow{3}{*}{ Vector maguibsos scurts per minus } & $72.1002+17,5995$ & $72.523 .3 \times 15.938 .1$ & $73,781.1+17,0150$ & $68.003 .7 \times 16,250.8$ \\
\hline & $19885=4596$ & $18522+421.9$ & $2002.6=4446$ & $1695.8+3924$ \\
\hline & Hormal Waizht $(a=33)$ & Overweiagtiotese $(\theta-29)$ & Fit $(a-46)$ & Gafil $(a=15)$ \\
\hline Ptrisica finess & $n=31$ & $n=29$ & $\pi=46$ & $n=15$ \\
\hline Nosdiate $\mathrm{VO}_{\min }\left(\mathrm{L}^{-1 \mathrm{~min}^{-1}}\right)$ & $3.48+1.21$ & $3.20=0.90$ & $3.51=0.94$ & $286 \pm 006 \cdots$ \\
\hline 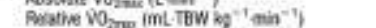 & $574: 127$ & $389+113^{*}$ & $544+12.1$ & $309=83^{\circ}$ \\
\hline 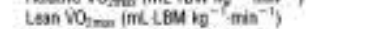 & $755=10.9$ & $68.1+163$ & $77.4 \div 99$ & $56.5 \times 7.6^{\circ}$ \\
\hline Rh Pesk foussle per mintes & $493=5.9$ & $468+8.1$ & $487+6.5$ & $43.0+89^{-4}$ \\
\hline HA quarcis sece & $8.42 \div 1.56$ & $10.12 \times 2.46^{*}$ & $9.68 \div 1.54$ & $1065=309 \cdots$ \\
\hline HA recoenry shos & $-18.87=8.48$ & $-15.49 \times 7.49$ & $-1806=755$ & $-1449+865$ \\
\hline mo & $1.06 \times 0.07$ & $1.05+0.06$ & $180+0.07$ & $103 \times 0 . \cdots$ \\
\hline O. puls imk por beas? & $17.5+5.2$ & $172+5.1$ & $17.8+5.6$ & $16.0+55$ \\
\hline Tima ve ediasstion (min) & $13.4=2.4$ & $102+24^{\circ}$ & $130+2.4$ & $91+24^{\circ}$ \\
\hline Evercise volanoss & $x=31$ & $\theta=29$ & $x=46$ & $A=15$ \\
\hline Peak RFE Quriso Vohar $(0-19)$ & $22=1.1$ & $90+1.7$ & $20=15$ & $21=12$ \\
\hline HR $=$ (S) & $97.3=8.2$ & $96.6 \pm 13.7$ & $980 \times 92$ & $92.0=15.2$ \\
\hline Experimsital pain & $x=32$ & $0=29$ & $n \geqslant 45$ & $n=15$ \\
\hline 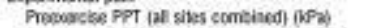 & $390.3=2013$ & $425.7+273.9$ & $379.9+207.7$ & $468.5+311.3$ \\
\hline 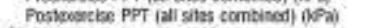 & $411.4=235.7$ & $462.3+314.3$ & $414.6 \pm 2383$ & $502.2 \div 363.4$ \\
\hline \multirow{2}{*}{\multicolumn{5}{|c|}{ MPQ }} \\
\hline & & & & \\
\hline MPO flobal, 0-78; & $4.4 \mathrm{a}=6.20$ & $3.76+3.72$ & $4.74 \pm 8.29$ & $227 \pm 381$ \\
\hline MPO $(P P L, 0-5)$ & $0.02+0.58$ & $0.4 \mathrm{a}+0.74$ & $0.83 \div 0.92$ & $0.53=0.74$ \\
\hline MPO IVAS $0-10 \mathrm{~cm}$ & $1.13=1.42$ & $0.40 .1 .11 \cdots$ & $0.89+1.4$ & $0.44 \div 0.67$ \\
\hline \multicolumn{5}{|l|}{ PCS } \\
\hline PCS-C tood, (0-52) & $16.39+10.22$ & $17.07 \div 10.04$ & $16.20+10.11$ & $10.67 \pm 10.21$ \\
\hline PCS P itoes, 0-52) & $13.52+9.65$ & $18.00 \pm 10.85$ & $14.78+9.74$ & $1937+10.20$ \\
\hline PCS.P IIragrificasion, O-121 & $2.70=2.00$ & $3.34 \pm 2.48$ & $2.83+2.16$ & $3.67+250$ \\
\hline PCS.P (ruminstho, $0-16$ ) & $6.52=3.51$ & $8.72 \pm 4.11 \cdots$ & $7.24 \pm 3.97$ & $8.90=3.65$ \\
\hline PCS.P inepessness, 0-24) & $4.30=4.20$ & $5.60+5.23$ & $4.72+4.47$ & $6.40 \div 5.50$ \\
\hline \multicolumn{5}{|l|}{ pedsol $(21,0-100)$. } \\
\hline Pedsol chive incait & $84.3+0.3$ & $843 \pm 11.8$ & $36.1 \div 83$ & $78.9+12.2 \cdots+$ \\
\hline Petsol chlo (Dhyseal) & $361=10.1$ & $848 \pm 11.9$ & $07.4 \pm 10.4$ & $800 \pm 11,4 \cdots$ \\
\hline Petsol chis estecis) & $91.5: 103$ & $68.8 \pm 133$ & $92 t+10.0$ & $84.0=15.0 \cdots$ \\
\hline Petsol chid iemofionali & $308>13.4$ & $816+181$ & $82.5 \div 14.1$ & $76.3 \times 19.8$ \\
\hline Predsol child (sctodi) & $77.7 \times 129$ & $51.7+15.3$ & $81.6+12.2$ & $74.7 \times 17.9$ \\
\hline Pedsol child ipeychesocis beath summany & $833+9.7$ & $840 \pm 140$ & $854=100$ & $78.3+15.7$ \\
\hline Pedsol parent thatil & $888=10.3$ & $827+185$ & $892=10.3$ & $754+21,6 \cdots$ \\
\hline Prodsol parent \{physices? & $923: 82$ & $60.4+210^{*} \cdots$ & $923+87$ & $74.8 \div 25.0 \cdots$ \\
\hline Pedsol panent (sodal) & $941+97$ & $86.21+202$ & $935+10.3$ & $90.3 \div 24.8$ \\
\hline Pedsoc parent fermotonalt & $820=17.1$ & $514 \pm 202$ & $83.8+16.4$ & $74.7+23.5$ \\
\hline Pedsol parent tschoul & $848=167$ & $79.5 \pm 200$ & $857=15.8$ & $720+22.5$ \\
\hline Pdosol parent iposchosocix heath sumnay) & $37.0=125$ & $824+182$ & $37,6=120$ & $75.7 \pm 21.5$ \\
\hline
\end{tabular}

\section{Table 1}

Self-reported physical activity (PAQ) was similar between the normal-weight and overweight/obese adolescents (Table 1); however, PAQ was inversely correlated with total body fat $z$-score $(r=-0.263$, $P=0.04)$, so that adolescents with more body fat reported less physical activity. On the basis of accelerometry $(n=46)$, normal-

Medicine \& Science in Sports \& Exercise, Vol 47, No. 11 (November 2015): pg. 2431-2440. DOI. This article is (C) American College of Sports Medicine and permission has been granted for this version to appear in e-Publications@Marquette. American College of Sports Medicine does not grant permission for this article to be further copied/distributed or hosted elsewhere without the express permission from American College of Sports Medicine. 
weight and overweight/obese adolescents had similar physical activity levels with the exception of average length of sedentary bouts (min) where overweight/obese adolescents had longer sedentary time ( $P=$ 0.005).

Overweight/obese adolescents had lower relative $\mathrm{V} \cdot \mathrm{O}_{2 \max }$ $\left(\mathrm{mL} \cdot \mathrm{kg}^{-1} \cdot \mathrm{min}^{-1}\right)(\mathrm{P}<0.0001)$ and decreased time to exhaustion during the aerobic capacity test $(P<0.0001)$ compared with the normalweight adolescents (Table 1 ). Absolute $\mathrm{V} \cdot \mathrm{O}_{2 \max }$ and lean $\mathrm{V} \cdot \mathrm{O}_{2 \max }$ were the same between the weight groups.

For the pain measures, baseline (preexercise) PPT were similar between the two weight groups. Furthermore, clinical pain and quality of life were similar between the weight groups, with the exception of current pain intensity (MPQ visual analog scale (VAS), $P=0.05$ ) where normal-weight adolescents reported higher pain than the overweight/obese adolescents. In addition, parent's perspective for physical quality of life $(P=0.04)$ was higher in the normal-weight group and pain catastrophizing rumination $(P=0.03)$ was lower in the normal-weight group in contrast to that in the overweight/obese group (Table 1).

\section{Baseline Measures in Fit versus Unfit Adolescents}

On the basis of the FitnessGram performance standards using relative $\mathrm{V} \cdot \mathrm{O}_{2 \max }$, adolescents were classified as fit $(\mathrm{n}=46 ; 30$ normal weight and 16 overweight/obese) or unfit ( $n=15$; two of normal weight and 13 overweight/obese) (Table 1). The two groups differed in that fit adolescents had higher absolute $V \cdot O_{2 \max }(P=0.02)$, lean $V \cdot O_{2 \max }(P<0.0001)$, peak RR $(P=0.002)$, and time to exhaustion ( $P$ $<0.0001$ (Table 1) than unfit adolescents. The fit group also had higher self-reported physical activity $(P=0.03)$, lower resting HR ( $P$ $<0.0001)$, and lower resting systolic pressure $(P=0.03)$ and diastolic pressure $(P=0.03)$. On the basis of ActiGraph monitoring, fit and unfit adolescents had similar physical activity levels, with the exception of average length of sedentary bouts (min) where fit adolescents had shorter sedentary time $(P=0.018)$. With respect to body composition and weight status, fit adolescents compared with unfit adolescents had lower BMI z-scores, body fat percentage, total body fat z-scores, fat mass, android fat, and gynoid fat (all $\mathrm{P}<$ 0.0001 ) but similar lean body mass. 
Specific to pain measures, baseline (preexercise) PPT were similar between the fit and unfit adolescents (Table 1 ). There were no differences between the fit and unfit group for the adolescent's clinical pain (MPQ) and the child's and parent's perspectives for pain catastrophizing $(P>0.05)$. Quality of life from the child's perspective (total quality of life $(P=0.02)$, physical functioning $(P=0.02)$, and social functioning ( $P=0.02$ ) were significantly higher in the fit adolescents compared with that in the unfit. Quality of life assessment from the parent's perspective (total quality of life $(P=0.02)$, physical functioning $(P=0.02)$, and school functioning $(P=0.01)$ were significantly higher in the fit adolescents (Table 1 ).

\section{Exercise Response}

All adolescents, regardless of weight status, met the American College of Sports Medicine criteria for the completion of a $\mathrm{V} \cdot \mathrm{O}_{2 \max }$ test, as follows: peak RPE $(9.1 \pm 1.4)$, RQ $(1.1 \pm 0.1)$, and $\mathrm{HR}_{\max }(96.5 \%$ $\pm 11.1 \%)$. There was no difference in peak RPE or percentage of $\mathrm{HR}_{\max }$ by weight status or physical fitness levels (Table 1). RQ differed between fitness levels in that unfit adolescents had lower RQ values than fit adolescents $(P=0.02)$ but did not differ with weight status (Table 1).

Perceived exertion increased during the aerobic capacity treadmill test (time, $\mathrm{P}<0.0001$ ). This increase was similar between fitness groups (time $\times$ fitness levels, $P>0.05$ ). In contrast, the increase in perceived exertion differed by weight status (time $x$ weight status, $P=0.04$ ). Post hoc analyses showed that perceived exertion at the end of stage 1 was greater for the overweight/obese adolescents $(1.8 \pm 2.0)$ compared with that for the normal-weight adolescents $(0.8 \pm 0.9, \mathrm{P}=0.04)$. There were no differences in perceived exertion at midpoint or termination of the treadmill.

\section{Experimental Pain after Quiet Rest and Exercise} Sessions

Pain thresholds increased after exercise (i.e., EIH) but were unchanged after quiet rest (trial $\times$ session, $P=0.02$ ) (Fig. 2). This response was similar across the sites assessed for pain (trial $x$ session $\times$ site, $P>0.05)$. 


\section{EIH: weight status and body composition}

\section{Figure 2}

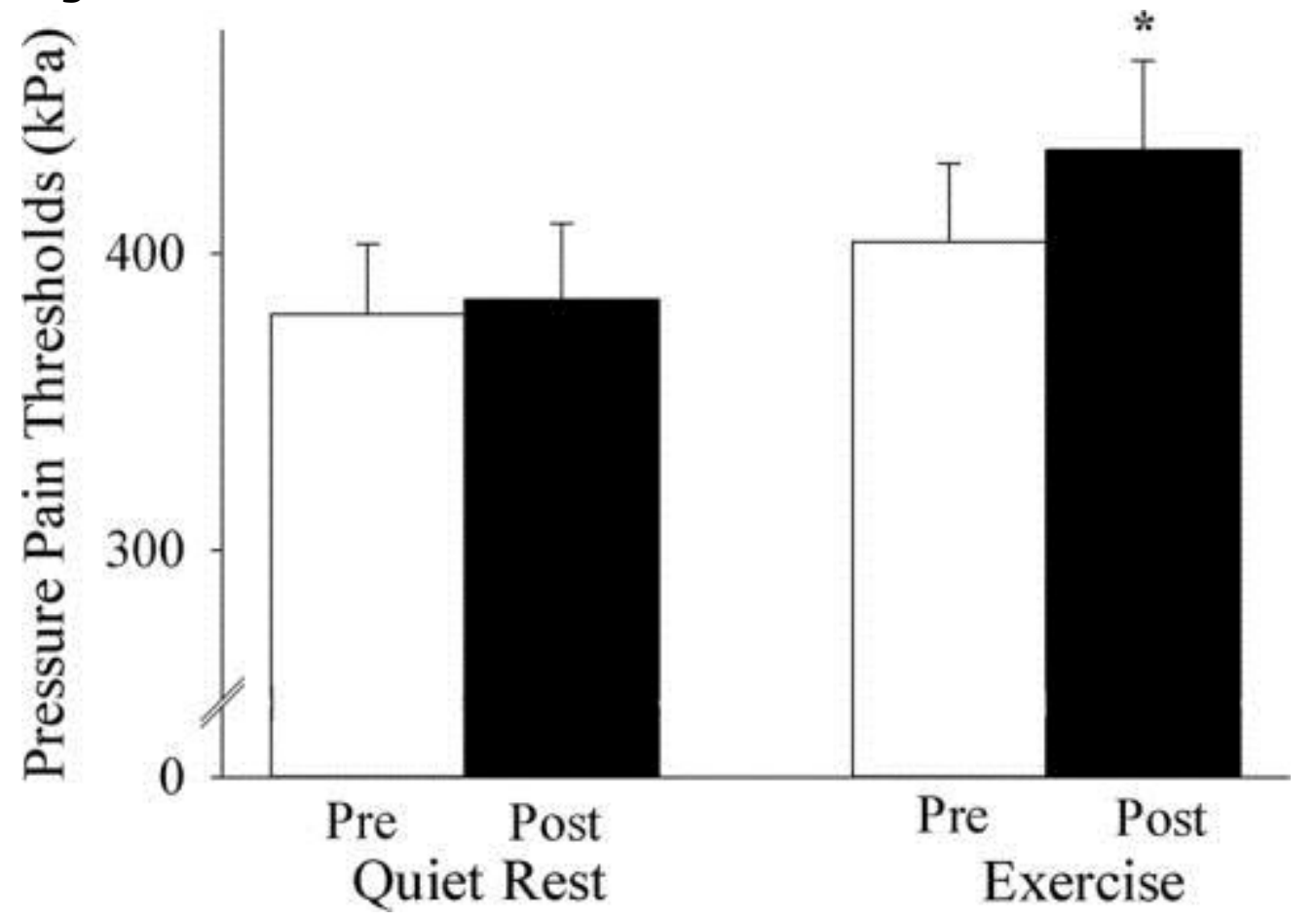

EIH was similar between the normal-weight and overweight/obese adolescents ( $P>0.05)$. Regardless of weight status, gynoid and android adolescents reported similar EIH (trial $\times$ android/gynoid, $P>0.05)$. Lean body mass $(\mathrm{kg})$ was weakly correlated with EIH ( $r=0.146, \mathrm{P}=0.05)$ (Fig. 3); adolescents with higher total body lean mass experienced greater $\mathrm{EIH}$, yet fat mass $(\mathrm{kg})$ was not correlated $(P>0.05)$.

EIH: physical fitness and activity

\section{Figure 3}

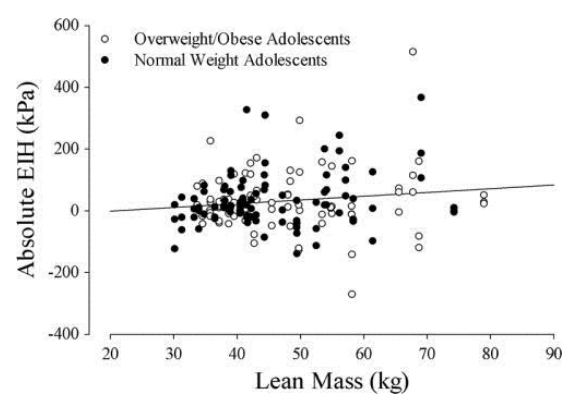

Medicine \& Science in Sports \& Exercise, Vol 47, No. 11 (November 2015): pg. 2431-2440. DOI. This article is (C) American College of Sports Medicine and permission has been granted for this version to appear in e-Publications@Marquette. American College of Sports Medicine does not grant permission for this article to be further copied/distributed or hosted elsewhere without the express permission from American College of Sports Medicine. 
Fit and unfit adolescents (based on relative $\mathrm{V} \cdot \mathrm{O}_{2 \max }$ ), reported similar EIH (trial $\times$ fitness, $P>0.05$ ). EIH was not correlated with relative $\mathrm{V} \cdot \mathrm{O}_{2 \max }$, absolute $\mathrm{V} \cdot \mathrm{O}_{2 \max }$, lean $\mathrm{V} \cdot \mathrm{O}_{2 \max }$, and peak $\mathrm{RR}$. Selfreported physical activity (PAQ) was not correlated with $\mathrm{EIH}$, but total sedentary bouts $(r=-0.189, P=0.03)$ from the ActiGraph monitors were weakly inversely correlated with $\mathrm{EIH}$; adolescents with greater sedentary time experienced less EIH. Furthermore, adolescents at risk for metabolic syndrome ( $n=38 ; 19$ normal weight and 19 overweight/obese) reported similar $\mathrm{EIH}$ compared with those not at risk for metabolic syndrome $(n=24 ; 14$ normal weight and 10 overweight/obese).

\section{EIH: clinical pain and quality of life}

Clinical pain measured with the MPQ was not correlated with EIH. PedsQL Child/Teen physical functioning was weakly correlated with EIH ( $r=0.149, \mathrm{P}=0.05)$; adolescents with greater physical functioning experienced greater EIH. PCS-C and PedsQL Parent were not associated with EIH ( $P>0.05)$.

\section{Discussion}

This study is the first to demonstrate that overweight/obese adolescents report reduced response to a pain stimulus after intense aerobic exercise, and this response was analogous to normal weight adolescents. The increase in pain threshold was similar across body sites (nailbed, deltoid muscle, quadriceps muscle) demonstrating a systemic response, which has important clinical implications and possibilities as a strategy for pain relief in adolescents. The decrease in pain perception throughout the body indicates that pain relief after exercise is not isolated to the exercising muscle. Thus, aerobic exercise has strong potential as a nonpharmacological pain management tool in adolescents regardless of their weight status.

Few studies have investigated the effect of weight status on EIH. Comstock et al. ${ }^{5}$ showed that after a single exercise session of moderately heavy resistance training, lean and obese men reported similar levels of soreness and fatigue; pain perception was not specifically addressed in this study. Most studies that focus on overweight/obese individuals tend to focus on changes in healthrelated quality of life in conjunction with weight loss. Our results specifically address the role of exercise in decreasing pain perception 
in overweight/obese adolescents and parallel other findings that have shown that exercise gains extend beyond weight loss. ${ }^{35}$ For individuals with knee osteoarthritis, decreasing body fat and increasing physical activity were more important than weight loss for symptomatic relief. ${ }^{35}$ Similarly, in the current study, EIH was weakly associated with lean body mass but not total body mass and lean mass was similar between the weight groups. For individuals who are overweight/obese, the benefits of exercise are manifold and significant improvements in health may occur independent of weight loss. 32

When the adolescents were categorized on the basis of physical fitness, EIH was similar between the fit and unfit groups. In addition, self-reported physical activity was not associated with EIH. Previously, we have shown that self-reported physical activity was not associated with pain relief after isometric contractions in young and older healthy adults. ${ }^{23}$ In contrast, there are some reports that inactive individuals may not experience the same benefits with exercise and may even report increase in pain; ${ }^{38}$ our data concurs that inactive adolescents with more sedentary bouts experience less pain reduction after exercise. However, when adolescents were identified as being "at risk" for metabolic syndrome using published cutoff scores for selfreported physical activity (PAQ), ${ }^{41}$ similar EIH occurred between the adolescent groups "at risk" and "no risk."

\section{Weight status}

Adolescents with higher BMI levels were categorized in the overweight/obese category, with most of these adolescents designated as obese. Of the 29 overweight/obese adolescents, 16 were classified as fit and 13 were classified as unfit. All of the weight status and body composition values, except lean body mass, were significantly different between the normal-weight and overweight/obese groups. In relation to physical fitness, relative $\mathrm{V} \cdot \mathrm{O}_{2 \max }\left(\mathrm{mL} \cdot \mathrm{kg}^{-1} \cdot \mathrm{min}^{-1}\right)$ was lower for the overweight/obese adolescents. There was no difference between the weight groups when lean $\mathrm{V} \cdot \mathrm{O}_{2 \max }$ (relative to lean body mass) was compared. Thus, the lower relative $\mathrm{V} \cdot \mathrm{O}_{2 \max }$ in overweight/obese adolescents was due to their elevated fat mass. Several studies have reported comparable results between obese and normal-weight participants; obese individuals tend to have lower relative $\mathrm{V} \cdot \mathrm{O}_{2 \max }$ when calculated 
relative to total body mass and similar levels as those of normalweight individuals when computed in relation to lean body mass. ${ }^{6,12,36}$ Overall, these results indicate that when total body mass is used as part of the physical fitness calculation, adolescents with greater mass have lower oxygen uptake. From a functional standpoint, they may have lower aerobic capacity when performing activities that necessitate movement of their body mass. ${ }^{12}$ In contrast, on the basis of the lean $\mathrm{V} \cdot \mathrm{O}_{2 \max }$, overweight/obese adolescents and normal-weight adolescents have similar maximal aerobic capacity.

Several investigators have shown that pain reports increase and quality of life decreases with increasing weight status. ${ }^{14,15,43}$ The adolescents in this study reported minimal to no current pain intensity. Surprisingly, the normal-weight adolescents reported slightly higher current pain intensity (VAS) compared with the overweight/obese adolescents; both pain intensity reports, however, were not clinically relevant. In contrast, Hainsworth et al. $\underline{14}$ found that $50 \%$ of obese youth (class 2 and 3 obesity) in a clinical setting reported current pain. In the current study, adolescents had a lower overall obese classification and were not participating in a clinical weight management program, which may help explain the differences in pain reports. Quality of life was also similar between the two weight groups and within normal limits for both the child and parent's perspectives. ${ }^{40}$ The only difference between the two weight groups was from the parent's perspective. Parents of overweight/obese adolescents reported lower physical functioning of their adolescent compared with parents of normal-weight adolescents. Although several studies have shown lower health-related quality of life with increasing weight status, ${ }^{43,44}$ this relation between quality of life and weight status tends to be more distinct in clinical populations than community-based sampling. ${ }^{43}$ Using community-based sampling, our results demonstrate that normal-weight and overweight/obese adolescents have minimal pain reports and comparable quality of life.

\section{Physical fitness}

When the adolescents were categorized on the basis of physical fitness levels, most of the adolescents $(75 \%)$ were determined to be in the healthy fitness zone (i.e., fit). All of the weight status and body composition measures, except lean body mass, were different between the fit and unfit groups. In relation to quality of life, fitness 
levels appeared to have a much bigger impact, both from the child and parent's perspectives, than weight status. Only one quality of life measure was different when comparing weight groups (parentphysical domain). When comparing fitness groups, however, six quality of life measures differed (three in the child and three in the parent). This finding emphasizes the need to view weight status and physical fitness as separate entities when addressing health outcomes. $\frac{12,21}{}$ Despite the differences in quality of life, experimental and clinical pain reports were similar for the fit and unfit adolescents.

As anticipated, self-reported physical activity and sedentary time (ActiGraph monitoring) were lower in the unfit than those in the fit adolescents. Self-reported physical activity level was then used to identify adolescents at risk for metabolic syndrome (boys, <2.9; girls, <2.7). ${ }^{41}$ The average physical activity for the unfit adolescents indicates that this group was at risk for metabolic syndrome. Surprisingly, the average for the fit adolescents (2.7) is borderline for metabolic syndrome. These results highlight the lack of physical activity in adolescents; only $8 \%$ of adolescents (12-19 yr) meet the US Department of Health and Human Services recommendations of 60 min of moderate-to-vigorous daily physical activity. ${ }^{26}$

Objective physical activity measurement is considered more valid and reliable than self-report. Unfortunately, this study demonstrates that considerable data can be lost in adolescent participants that compete in sporting activities because referees and/or coaches require the adolescents to remove the device. The missing data no doubt affect the results for moderate/vigorous physical activity levels with a greater effect between the fitness levels than that in weight groups. For example, of the 11 adolescent athletes that were told to remove the device, the missing data were evenly distributed between normal-weight $(n=6)$ and overweight/obese $(n=5)$ adolescents. In contrast, nine of the 11 adolescents were "fit" versus two who were "unfit." As a result, this may have contributed to the lack of difference in physical activity, specifically for moderate/vigorous activity between fitness levels. These results highlight the challenges in capturing wrist-based accelerometry in an active pediatric population. 
NOT THE PUBLISHED VERSION; this is the author's final, peer-reviewed manuscript. The published version may be accessed by following the link in the citation at the bottom of the page.

\section{Exercise response}

Although maximal aerobic exercise is not typically prescribed for pain management in adolescents, we chose this exercise dose because it is a measure of physical fitness and it allowed us to determine the $\mathrm{EIH}$ at maximal dose. It also allowed us to investigate whether adolescents could tolerate maximal aerobic exercise. One reason for the lack of evidence on whether obese individuals experience $\mathrm{EIH}$ is the concern that they cannot tolerate regular exercise and are at higher risk for injury. ${ }^{25}$ In this study, perceived exertion was similar between the normal-weight and overweight adolescents except at the initiation of the treadmill test when obese adolescents reported slightly higher perceived exertion. Furthermore, all of the adolescents tolerated and met the American College of Sports Medicine criteria for termination of the $\mathrm{V} \cdot \mathrm{O}_{2 \max }$ test, and none of the adolescents experienced any contraindications for early termination. This exercise protocol is in line with Expert Committee recommendations for the prevention and treatment of child and adolescent overweight/obesity by promoting moderate-to-vigorous physical activity for at least $60 \mathrm{~min} \cdot \mathrm{d}^{-1} \cdot 1$ Although maximal $\mathrm{V} \cdot \mathrm{O}_{2}$ testing is not typically used as an exercise stimulus in the clinic, our results indicate that adolescents of different weight status tolerate and experience pain relief after maximal aerobic exercise.

Taking into account the differences in the $\mathrm{V} \cdot \mathrm{O}_{2 \max }$ in relation to total body mass and lean mass, exercise tolerance could be affected on the basis of the degree of body mass movement (e.g., running vs cycling). Because of the shorter time to exhaustion and lower $\mathrm{V} \cdot \mathrm{O}_{2 \max }$ relative to total body mass, obese adolescents may have more difficulty in the performance of weight-bearing activities, $\underline{12}$ although this was not reflected in their perceived exertion at the midpoint or termination of the treadmill protocol. Thus, different $\mathrm{V} \cdot \mathrm{O}_{2 \max }$ calculations (total body mass vs lean mass) may be used to calculate physical fitness and help establish performance levels during weightbearing vs non-weight-bearing activities.

\section{Limitations}

Although this study helps lay the foundation for the prescription of maximal aerobic exercise in the management of pain, there are some limitations. First, the population sample should be expanded to

Medicine \& Science in Sports \& Exercise, Vol 47, No. 11 (November 2015): pg. 2431-2440. DOI. This article is @ American College of Sports Medicine and permission has been granted for this version to appear in e-Publications@Marquette. American College of Sports Medicine does not grant permission for this article to be further copied/distributed or hosted elsewhere without the express permission from American College of Sports Medicine. 
include more distinct weight groups (overweight through class 3 obesity). These distinctions would help identify whether any changes in pain at rest and after exercise occur similarly at each level of weigh status. Second, physical activity measured by self-report has the potential for inaccuracies, as both over- and underreporting have been described in youth. However, objective physical activity monitoring may also be limited when adolescents participate in organized competitive sports. Third, an order effect may be present in this study's design, with the quiet rest condition always occurring before the exercise condition. Finally, we categorized the adolescents as fit or unfit on the basis of their relative $\mathrm{V} \cdot \mathrm{O}_{2 \max }\left(\mathrm{mL} \cdot \mathrm{kg}^{-1} \cdot \mathrm{min}^{-1}\right)$. Although this is the primary calculation used in determining physical fitness levels, excess adiposity in obese individuals may result in lower perceived physical fitness levels than if the $\mathrm{V} \cdot \mathrm{O}_{2 \max }$ was based on lean mass. Despite this equivocality, there are no standardized data to use $\mathrm{V} \cdot \mathrm{O}_{2 \max }$ per lean mass as a marker for physical fitness.

\section{Conclusions}

These results significantly add to the literature by providing much needed evidence in the prescription of therapeutic exercise as a pain management tool for adolescents of varying weight and fitness levels. Both normal-weight and overweight/obese adolescents experienced similar levels of EIH after maximal aerobic exercise. In addition, physical fitness levels did not influence the magnitude of $\mathrm{EIH}$, but sedentary time was associated with EIH. Nevertheless, physical fitness levels may be more important than weight status when determining quality of life in adolescents. When measuring physical fitness, the influence of total body mass and lean mass on $\mathrm{V} \cdot \mathrm{O}_{2 \max }$ should be assessed for a broader understanding of physical fitness levels and implications for weight-bearing and non-weightbearing activities. Additional pediatric research is warranted in identifying the effect of weight status on pain perception at rest and after exercise with multiple stages of obesity and community-based versus clinically based populations.

This project was supported by the following: 1) American Association of University Women-American Fellowship (S. S.), 2) Foundation for Physical Therapy-Promotion of Doctoral Scholarships I and II (S. S.), 3) Marquette University President's Council-Raynor Fellowship (S. S.), and 4) National Center for Advancing Translational Sciences, National Institutes of Health, through grant number 8 UL1TR000055 (S. S., M. D., S. H., M. H. B.). 
NOT THE PUBLISHED VERSION; this is the author's final, peer-reviewed manuscript. The published version may be accessed by following the link in the citation at the bottom of the page.

The authors declare no conflicts of interest. The results of the present study do not constitute endorsement by the American College of Sports Medicine.

\section{References}

${ }^{1}$ Barlow SE. Expert committee recommendations regarding the prevention, assessment, and treatment of child and adolescent overweight and obesity: summary report. Pediatrics. 2007; 120(4 Suppl): S164-92.

2 Birnie KA, Caes L, Wilson AC, Williams SE, Chambers CT. A practical guide and perspectives on the use of experimental pain modalities with children and adolescents. Pain Manag. 2014; 4(2): 97-111.

${ }^{3}$ Cain KL, Sallis JF, Conway TL, Van Dyck D, Calhoon L. Using accelerometers in youth physical activity studies: a review of methods. J Phys Act Health. 2013; 10(3): 437-50.

${ }^{4}$ Choi L, Liu Z, Matthews CE, Buchowski MS. Validation of accelerometer wear and nonwear time classification algorithm. Med Sci Sports Exerc. 2011; 43(2): 357-64.

${ }^{5}$ Comstock BA, Thomas GA, Dunn-Lewis C, et al. Effects of acute resistance exercise on muscle damage and perceptual measures between men who are lean and obese. J Strength Cond Res. 2013; 27(12): 348894.

${ }^{6}$ Cooper DM, Poage J, Barstow TJ, Springer C. Are obese children truly unfit? Minimizing the confounding effect of body size on the exercise response. J Pediatr. 1990; 116(2): 223-30.

7 Dodet $P$, Perrot S, Auvergne L, et al. Sensory impairment in obese patients? Sensitivity and pain detection thresholds for electrical stimulation after surgery-induced weight loss, and comparison with a nonobese population. Clin J Pain. 2013; 29(1): 43-9.

${ }^{8}$ Ehrman JK, American College of Sports Medicine. ACSM's Guidelines for Exercise Testing and Prescription. 9th ed. Philadelphia (PA): Wolters Kluwer Health/Lippincott Williams \& Wilkins; 2014: 112-5.

${ }^{9}$ Ekblom O, Nyberg G, Bak EE, Ekelund U, Marcus C. Validity and comparability of a wrist-worn accelerometer in children. J Phys Act Health. 2012; 9(3): 389-93.

${ }^{10}$ Fakhouri TH, Hughes JP, Brody DJ, Kit BK, Ogden CL. Physical activity and screen-time viewing among elementary school-aged children in the United States from 2009 to 2010. JAMA Pediatr. 2013; 167(3): 2239.

Medicine \& Science in Sports \& Exercise, Vol 47, No. 11 (November 2015): pg. 2431-2440. DOI. This article is @ American College of Sports Medicine and permission has been granted for this version to appear in e-Publications@Marquette. American College of Sports Medicine does not grant permission for this article to be further copied/distributed or hosted elsewhere without the express permission from American College of Sports Medicine. 
NOT THE PUBLISHED VERSION; this is the author's final, peer-reviewed manuscript. The published version may be accessed by following the link in the citation at the bottom of the page.

${ }^{11}$ Freedson $\mathrm{P}$, Pober D, Janz KF. Calibration of accelerometer output for children. Med Sci Sports Exerc. 2005; 37(11 Suppl): S523-30.

12 Goran M, Fields DA, Hunter GR, Herd SL, Weinsier RL. Total body fat does not influence maximal aerobic capacity. Int J Obes Relat Metab Disord. 2000; 24(7): 841-8.

13 Groenewald CB, Essner BS, Wright D, Fesinmeyer MD, Palermo TM. The economic costs of chronic pain among a cohort of treatment-seeking adolescents in the United States. J Pain. 2014; 15(9): 925-33.

${ }^{14}$ Hainsworth KR, Miller LA, Stolzman SC, et al. Pain as a comorbidity of pediatric obesity. Infant Child Adolesc Nutr. 2012; 4(5): 315-20.

15 Heo M, Allison DB, Faith MS, Zhu S, Fontaine KR. Obesity and quality of life: mediating effects of pain and comorbidities. Obes Res. 2003; 11(2): 209-16.

16 Hogeweg JA, Kuis W, Oostendorp RA, Helders PJ. The influence of site of stimulation, age, and gender on pain threshold in healthy children. Phys Ther. 1996; 76(12): 1331-9.

17 Huguet A, Miro J. The severity of chronic pediatric pain: an epidemiological study. J Pain. 2008; 9(3): 226-36.

${ }^{18}$ Kelly TL, Wilson KE, Heymsfield SB. Dual energy x-ray absorptiometry body composition reference values from NHANES. PLoS One. 2009; 4(9): e7038.

${ }^{19}$ Koltyn KF. Exercise-induced hypoalgesia and intensity of exercise. Sports Med. 2002; 32(8): 477-87.

${ }^{20}$ Kosek E, Lundberg L. Segmental and plurisegmental modulation of pressure pain thresholds during static muscle contractions in healthy individuals. Eur J Pain. 2003; 7(3): 251-8.

${ }^{21}$ Krachler B, Savonen K, Komulainen P, Hassinen M, Lakka TA, Rauramaa R. Cardiopulmonary fitness is a function of lean mass, not total body weight: the DR's EXTRA study. Eur J Prev Cardiol. 2015; 22: 1171-9.

22 Ladabaum U, Mannalithara A, Myer PA, Singh G. Obesity, abdominal obesity, physical activity, and caloric intake in US Adults: 1988 to 2010. Am J Med. 2014; 127(8): 717-27.

${ }^{23}$ Lemley KJ, Hunter SK, Bement MK. Conditioned pain modulation predicts exercise-induced hypoalgesia in healthy adults. Med Sci Sports Exerc. 2015; 47(1): 176-84.

${ }^{24}$ Lim CS, Mayer-Brown SJ, Clifford LM, Janicke DM. Pain is associated with physical activity and health-related quality of life in overweight and obese children. Child Health Care. 2014; 43(3): 186-202.

Medicine \& Science in Sports \& Exercise, Vol 47, No. 11 (November 2015): pg. 2431-2440. DOI. This article is (C) American College of Sports Medicine and permission has been granted for this version to appear in e-Publications@Marquette. American College of Sports Medicine does not grant permission for this article to be further copied/distributed or hosted elsewhere without the express permission from American College of Sports Medicine. 
NOT THE PUBLISHED VERSION; this is the author's final, peer-reviewed manuscript. The published version may be accessed by following the link in the citation at the bottom of the page.

25 McHugh MP. Oversized young athletes: a weighty concern. Br J Sports Med. 2010; 44(1): 45-9.

${ }^{26}$ Office of Disease Prevention and Health Promotion Web site [Internet]. Washington (DC). Physical Activity Guidelines for Americans, Chapter 3:Active Children and Adolescents [cited 2015 01/21]. Available from: http://www.health.gov/paguidelines/guidelines/chapter3.aspx.

27 Ogden CL, Carroll MD, Kit BK, Flegal KM. Prevalence of childhood and adult obesity in the United States, 2011-2012. JAMA. 2014; 311(8): 80614.

28 Pielech M, Ryan M, Logan D, Kaczynski K, White MT, Simons LE. Pain catastrophizing in children with chronic pain and their parents: proposed clinical reference points and re-examination of the Pain Catastrophizing Scale measure. Pain. 2014; 155(11): 2360-7.

29 Preedy V. Handbook of Anthropometry: Physical Measures of Human Form in Health and Disease. New York: Springer-Verlag. 2012: 29-48.

30 Price RC, Asenjo JF, Christou NV, Backman SB, Schweinhardt P. The role of excess subcutaneous fat in pain and sensory sensitivity in obesity. Eur J Pain. 2013; 17(9): 1316-26.

${ }^{31}$ Staud R. Abnormal endogenous pain modulation is a shared characteristic of many chronic pain conditions. Expert Rev Neurother. 2012; 12(5): 577-85.

32 Stolzman S, Hoeger Bement M. Inflammatory markers in pediatric obesity: health and physical activity implication. Infant Child Adolesc Nutr. 2012; 4(5): 297-302.

${ }^{33}$ Stone AA, Broderick JE. Obesity and pain are associated in the United States. Obesity (Silver Spring). 2012; 20(7): 1491-5.

34 The Cooper Institute. Fitnessgram/Activitygram Reference Guide. 4th ed. Dallas (TX): Internet Resource; 2013. pp. 63-71.

35 Toda Y, Toda T, Takemura S, Wada T, Morimoto T, Ogawa R. Change in body fat, but not body weight or metabolic correlates of obesity, is related to symptomatic relief of obese patients with knee osteoarthritis after a weight control program. J Rheumatol. 1998; 25(11): 2181-6.

36 Treuth MS, Figueroa-Colon R, Hunter GR, Weinsier RL, Butte NF, Goran MI. Energy expenditure and physical fitness in overweight vs nonoverweight prepubertal girls. Int J Obes Relat Metab Disord. 1998; 22(5): 440-7.

Medicine \& Science in Sports \& Exercise, Vol 47, No. 11 (November 2015): pg. 2431-2440. DOI. This article is @ American College of Sports Medicine and permission has been granted for this version to appear in e-Publications@Marquette. American College of Sports Medicine does not grant permission for this article to be further copied/distributed or hosted elsewhere without the express permission from American College of Sports Medicine. 
NOT THE PUBLISHED VERSION; this is the author's final, peer-reviewed manuscript. The published version may be accessed by following the link in the citation at the bottom of the page.

37 Trost SG, Loprinzi PD, Moore R, Pfeiffer KA. Comparison of accelerometer cut points for predicting activity intensity in youth. Med Sci Sports Exerc. $2011 ; 43(7)$ : 1360-8.

38 Umeda M, Corbin LW, Maluf KS. Examination of contraction-induced muscle pain as a behavioral correlate of physical activity in women with and without fibromyalgia. Disabil Rehabil. 2014; 20: 1-6.

39 Vaegter HB, Handberg G, Graven-Nielsen T. Similarities between exerciseinduced hypoalgesia and conditioned pain modulation in humans. Pain. 2014; 155(1): 158-67.

40 Varni JW, Limbers CA. The pediatric quality of life inventory: measuring pediatric health-related quality of life from the perspective of children and their parents. Pediatr Clin North Am. 2009; 56(4): 843-63.

41 Voss C, Ogunleye AA, Sandercock GR. Physical Activity Questionnaire for children and adolescents: English norms and cut-off points. Pediatr Int. 2013; 55(4): 498-507.

42 Washington RL, Bricker JT, Alpert BS, et al. Guidelines for exercise testing in the pediatric age group. From the Committee on Atherosclerosis and Hypertension in Children, Council on Cardiovascular Disease in the Young, the American Heart Association. Circulation. 1994; 90(4): 2166-79.

43 Williams J, Wake M, Hesketh K, Maher E, Waters E. Health-related quality of life of overweight and obese children. JAMA. 2005; 293(1): 70-6.

44 Zeller $\mathrm{MH}$, Modi AC. Predictors of health-related quality of life in obese youth. Obesity (Silver Spring). 2006; 14(1): 122-30.

Medicine \& Science in Sports \& Exercise, Vol 47, No. 11 (November 2015): pg. 2431-2440. DOI. This article is (C) American College of Sports Medicine and permission has been granted for this version to appear in e-Publications@Marquette. American College of Sports Medicine does not grant permission for this article to be further copied/distributed or hosted elsewhere without the express permission from American College of Sports Medicine. 